\title{
Ophthalmic Outcome in a Belgian Cohort of Cystinosis Patients Treated with a Compounded Preparation of Cysteamine Eye Drops: Retrospective Analysis
}

Freya Peeters - Catherine Cassiman - Karel Van Keer - Elena Levtchenko •

Koenraad Veys · Ingele Casteels

Received: September 1, 2019 / Published online: September 27, 2019

(C) The Author(s) 2019

\section{ABSTRACT}

Introduction: Treatment of the anterior segment problems in cystinosis is challenging as oral cysteamine is ineffective in the treatment of corneal problems because of its avascular structure. Although cysteamine eye drops have been formulated to counter this issue, the stability of cysteamine in these off-licensed formulations and treatment compliance are major problems. The aim of this retrospective study was to determine the efficacy of a compounded preparation of aqueous $0.5 \%$ cysteamine eye drops in the management of corneal complications of cystinosis.

Methods: Data of patients attending the multidisciplinary cystinosis clinic at the University Hospitals Leuven, Belgium between January 2015 and December 2018 were analyzed. All cystinosis patients were treated with the

Enhanced digital features To view enhanced digital features for this article go to https://doi.org/10.6084/ m9.figshare.9861677.

F. Peeters · C. Cassiman · K. Van Keer .

I. Casteels $(\square)$

Department of Ophthalmology, University

Hospitals, Leuven, Belgium

e-mail: ingele.casteels@uzleuven.be

E. Levtchenko $\cdot$ K. Veys

Department of Pediatrics, University Hospitals,

Leuven, Belgium compounded preparation of aqueous $0.5 \%$ cysteamine eye drops and oral cysteamine.

Results: A total of 12 patients were treated with the compounded preparation of aqueous $0.5 \%$ cysteamine eye drops, of whom $75 \%$ were aged $>18$ years $(n=9)$. The mean instillation frequency of the cysteamine eye drops was 3.3 drops/eye per day, and the mean number of hospital visits was two per year. All patients showed photophobia, > 30\% corneal infiltration, blepharospasm, eye pain and conjunctival hyperemia during the study period. None of these symptoms improved with treatment with aqueous compounded $0.5 \%$ cysteamine eye drops. The corneal cystine crystal score was $\geq 2$ in all patients at the last visit.

Conclusion: Treatment with the compounded preparation of aqueous $0.5 \%$ cysteamine eye drops, combined with oral cysteamine, was not effective in reducing corneal cystine crystal deposition and other ocular symptoms in these patients with cystinosis.

Funding: Recordati Rare Diseases.

Keywords: Aqueous $0.5 \%$ cysteamine eye drops; Compounded preparation; Corneal cystine infiltration; Cystinosis

\section{INTRODUCTION}

Cystinosis is a rare autosomal recessive disorder characterized by excessive intracellular cystine 
accumulation $[1,2]$. The incidence of cystinosis is approximately 1 in 100,000 to 1 in 200,000 live births $[1,3]$. Patients with cystinosis show bi-allelic mutations in the CTNS gene (17p13.2) which encodes for the lysosomal transporter protein cystinosin. The absence of cystinosin causes the accumulation of cystine in the lysosomes of cells where they are deposited as crystals [4]. Excessive deposition of cystine crystals causes tissue damage in multiple organs, including the kidney, eye, pancreas, liver, thyroid and muscles [3, 5].

Three clinical subtypes of cystinosis are recognized, namely, infantile/early onset nephropathic cystinosis (INC), juvenile/intermediate/ late-onset nephropathic cystinosis and ocular/ non-nephropathic cystinosis; these subtypes differ based on the age at diagnosis and severity of the disease $[1,3,6]$. INC is the most frequent type of cystinosis, accounting for up to $95 \%$ of cases; patients are generally healthy at birth and develop symptoms between 4 and 6 months of age [3]. INC is mainly characterized by renal Fanconi syndrome, and if left untreated it may lead to end-stage renal failure and death by 12 years of age [3]. Juvenile cystinosis is a less severe type of cystinosis and generally presents at a later age. Patients with ocular cystinosis show isolated accumulation of cystine crystals in the cornea without any systemic problems [4]. All three subtypes of cystinosis are characterized by the accumulation of cystine crystals in the cornea, leading to photophobia, keratopathies and corneal erosions [1, 3, 4, 6, 7]. Crystal deposition has also been identified in other ocular structures, including the conjunctiva, iris, choroid, ciliary body and optic nerve [8].

Cystinosis is generally treated with oral cysteamine, an amino-thiol that induces a thioldisulphide interchange reaction that generates cysteine and cysteine-cysteamine, two compounds that exit the lysosomes using alternate transporter systems [5, 6, 9]. Long-term cysteamine therapy is reported to effectively alleviate the symptoms of cystinosis and is associated with an increased life expectancy in patients who initiate the treatment early and show good treatment adherence [10]. However, oral cysteamine is not effective in the treatment of all ocular manifestations of cystinosis as it does not reach the cornea in the required concentrations due to the absence of a vascular supply $[5,11]$. Cysteamine eye drops have been formulated to address this issue and are reported to improve the corneal symptoms of cystinosis [7, 12-15]. It is recommended that the drops be used frequently (1 drop/eye every waking hour) to control and diminish corneal crystal deposition [16].

Here we report a case series of 12 patients with INC who were treated with aqueous $0.5 \%$ cysteamine eye drops, in addition to peroral cysteamine, at the University Hospitals Leuven, Belgium.

\section{METHODS}

This retrospective analysis was based on the clinical data of patients with cystinosis who attended a multidisciplinary cystinosis clinic at the University Hospitals Leuven, Belgium between January 2015 and December 2018. Patients attending the multidisciplinary cystinosis clinic were able to see the nephrologist, ophthalmologist and other specialists relevant to their case on the same day, and all identified patients with cystinosis were offered an ophthalmological examination twice a year. The primary focus of the study was to determine both corneal cystine crystal accumulation in patients with cystinosis and the efficacy of cysteamine eye drops in the management of corneal cystine accumulation in these patients. All patients included in this study were examined for cystine accumulation using the methodology described by Pinxten et al. [17].

All data used in this retrospective analysis had been previously acquired for other purposes. According to the Ethical Committee of the University Hospitals Leuven, no further ethical review or consent was required.

\section{Patient History}

A full medical history of each patient was obtained at first visit. The information collected included the clinical type of cystinosis, the mutation responsible for cystinosis, family 
history and family/personal history of medications used for the treatment of cystinosis, including the use of systemic and topical cysteamine. The patient's personal experience of visual acuity, degree of pain and blepharospasm were also recorded along with self- and clinician-assessed photophobia, as determined using the photophobia scaling system of Liang and colleagues [18]. Clinicians also assessed the degree of pain using the visual analog scale (VAS), along with visual acuity using the Snellen's and Parinaud's charts and blepharospasm by visual inspection.

\section{Treatment}

At the time of diagnosis of the systemic disease, all patients were advised to start using aqueous $0.5 \%$ cysteamine eye drops $\geq 6$ times in each eye daily. The cysteamine drops used by patients were prepared by the University Hospitals Leuven pharmacy and contained cysteamine hydrochloride $50 \mathrm{mg}$ in an aqueous solution with monopotassium phosphate, disodium phosphate, benzalkonium chloride and ascorbic acid (Table 1). Patients were advised to keep the drops in the freezer until required. Once thawed, they could only be stored in the fridge for up to 1 month.

Table 1 Composition of aqueous $0.5 \%$ cysteamine eye drops used

\begin{tabular}{ll}
\hline Ingredient & Quantity \\
\hline Monopotassium phosphate anhydrous & $11.6 \mathrm{mg}$ \\
Sodium chloride & $30 \mathrm{mg}$ \\
Disodium phosphate anhydrous & $82.50 \mathrm{mg}$ \\
Cysteamine hydrochloride & $50 \mathrm{mg}$ \\
Benzalkonium chloride & $1 \mathrm{mg}$ \\
Ascorbic acid & $2 \mathrm{mg}$ \\
Distilled water & q.s. $10 \mathrm{ml}$ \\
\hline
\end{tabular}

Storage conditions: Keep in the freezer; can be kept in the fridge for 1 month after thawing

qs Quantity sufficient
At the time of the investigation, all patients, with one exception, were using oral cysteamine in addition to being started on topical cysteamine drops. Data on dosage and cystine concentration in white blood cells are given in Table 2.

\section{Assessments}

Patients attending the clinic were encouraged to continue regular use of the cysteamine eye drops and were assessed twice each year for accumulation of corneal cystine crystals and other ophthalmologic manifestations of cystinosis. At each ophthalmological examination slit lamp biomicroscopy was performed for the examination of the conjunctiva, cornea, iris and lens, and visual acuity was assessed for each eye separately and both eyes together by measuring the best-corrected visual acuity (BCVA) at a distance (6 m, using the Snellen chart) and for near vision (using the Parinaud chart).

Conjunctiva was assessed for the presence of cystine crystals, and the level of bulbar conjunctival hyperemia was quantified using the Cornea and Contact Lens Research Unit (CCLRU) grading scale [19]. The degree of cystine accumulation in the cornea was determined by scoring visible corneal cystine crystals [corneal cystine crystal score (CCCS)] using slitlamp photographs with increasing cystine crystal densities (0.00-3.00) [7]. Corneal fluorescein staining was also evaluated, and any evidence of filamentous/band keratopathy was recorded with special attention to complications, such as iris transillumination, crystal deposition and posterior synechiae.

Anterior segment optical coherence tomography was used to quantify the depth of cystine crystal deposition in the cornea, as in vivo confocal microscopy was not available at the clinic at the time of the study. Fundoscopy was performed after dilation of each eye with $0.5 \%$ tropicamide and 10\% phenylephrine, and the optic disc and macula were observed for crystals. The crystals on the retinal surface were also studied, along with any depigmentation or alterations of the retinal pigment and epithelium. Fundus examination was documented 
using the ultra-wide field fundus camera (Optos Inc., Marlborough, MA, USA), and the macula was assessed by posterior segment optical coherence tomography (PS-OCT).

Data on each individual patient were analyzed, and median and range for the patient population were calculated for the most important parameters. No further statistical analysis was performed.

\section{RESULTS}

Since January 2015, 13 patients with confirmed cystinosis attended the multidisciplinary clinic. All patients were treated with compounded aqueous $0.5 \%$ cysteamine eye drops $(6 \times$ each day) and oral cysteamine since the confirmation of their diagnosis. All patients had planned follow-up visits at the hospital. One patient died before the first follow-up visit during the study period. Results are therefore available for 12 patients.

The characteristics of the 12 patients are shown in Tables 2 and 3 with respect to the anterior segment- and posterior segment-related manifestations, respectively. The majority $(75 \%)$ of patients were aged $>18$ years $(n=9)$. The presence of the CTNS gene mutation responsible for the disease was noted in each patient. Clinically assessed photophobia was mainly Grade $3(n=5)$, blepharospasm was generally mild $(n=6)$ and conjunctival injection was mainly Grade $1 \quad(n=5)$. Of those patients aged $\geq 32$ years $(n=4)$, three showed Grade 5 photophobia and severe blepharospasm. All four of these patients had ocular complications, one patient had corneal thinning and another one macular edema and cystine crystal deposition. The majority of patients had a VAS score of $<5(n=9)$ and $50 \%$ of patients had a Snellen's BCVA of $\geq 1.0$ (logMAR 0) (Tables 2, 3, 4).

The mean number of hospital visits for patients included in the study was two per year, and the mean patient-reported instillation frequency was 3.3 drops/eye per day (Table 2). At the end of the study, the average duration of topical treatment with the compounded preparation of aqueous $0.5 \%$ cysteamine eye drops was 30 years in patients aged $>18$ years and 8 years in patients aged $<18$ years. One patient, however, never used the prescribed drops. Despite treatment with compounded aqueous $0.5 \%$ cysteamine eye drops, the Snellen BCVA score decreased with age, and $22 \%$ of patients aged $>18$ years showed a Snellen score of $<20 / 200$ at the last visit (Table 4 ). A higher CCCS score was seen in patients aged $>18$ years compared with those aged $<12$ years ( 3 vs. 2 ; Table 2). All patients had a CCCS of $\geq 2$ at the end of the study (Table 2).

Patients in all age groups showed a minimum of 30\% corneal cystine infiltration during the study; the thickness of crystals was at least $60 \%$ in all age groups despite treatment with $0.5 \%$ cysteamine eye drops (Table 4 ). All patients aged $>18$ years of age showed photophobia (self-reported or clinician-assessed) compared with $67 \%$ of patients in the younger age group (Table 4). Blepharospasm, eye pain, and conjunctival hyperemia were also observed in all patients during the study period; none of these symptoms improved in a clinically relevant way with aqueous $0.5 \%$ cysteamine eye drops.

\section{DISCUSSION}

This retrospective study of ophthalmological data of 12 cystinosis patients treated at the University Hospitals Leuven showed that, despite receiving treatment with a compounded preparation of aqueous $0.5 \%$ cysteamine eye drops combined with oral cysteamine, patients developed progressive corneal cystine deposition and keratopathy.

Corneal cystine crystals in patients with cystinosis appear as highly reflective needles in the epithelium, stroma and endothelium [8]. These crystals can be identified as early as at 12 months of age in patients with INC and are clearly visible by 18 months of age [4]. The crystals tend to accumulate first in the peripheral anterior corneal layers and later progress to the posterior cornea and towards the center, eventually filling the whole cornea with cystine crystals. These crystals are visible with the naked eye as a hazy layer once the entire cornea 


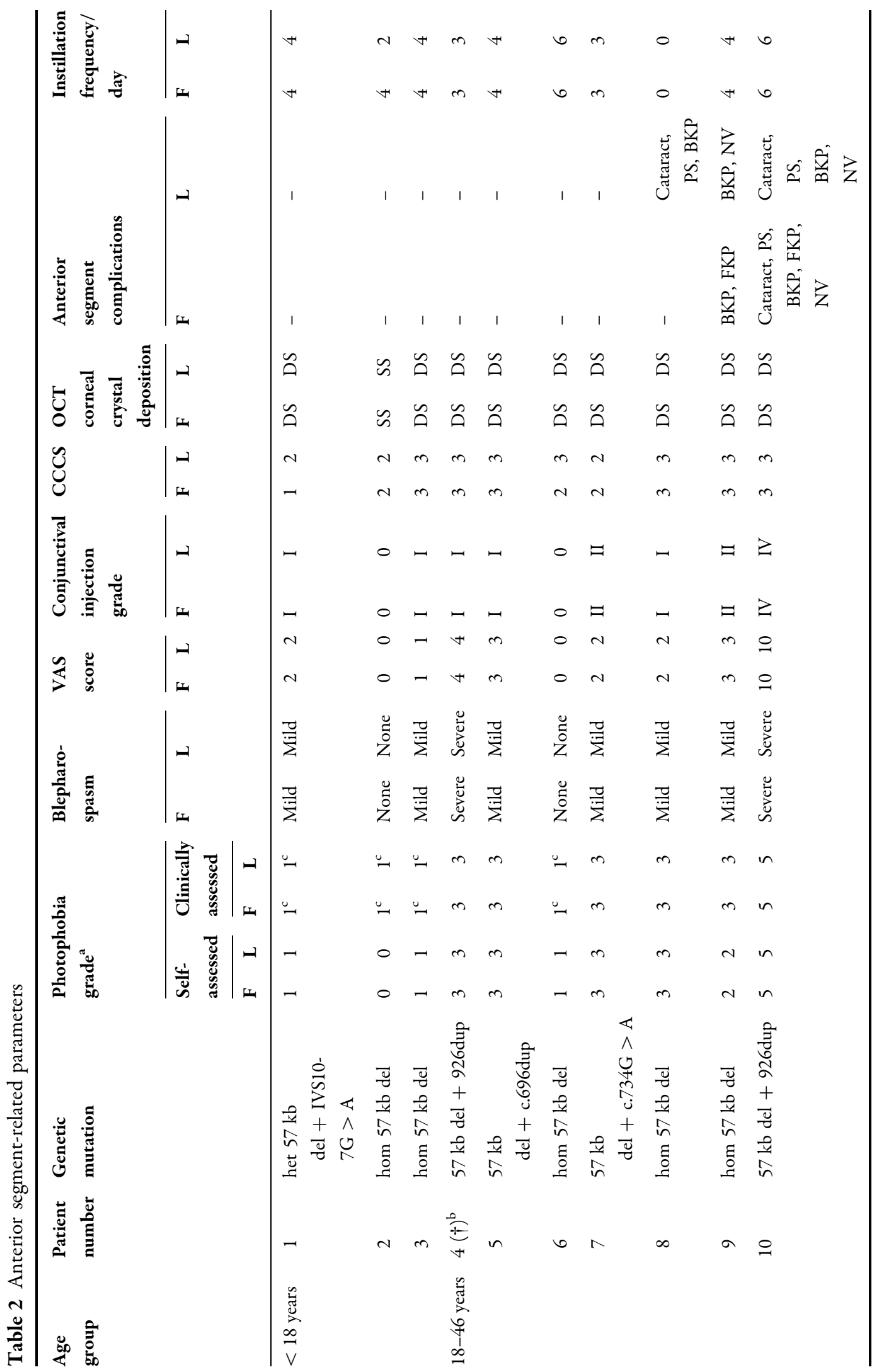


628

Ophthalmol Ther (2019) 8:623-633

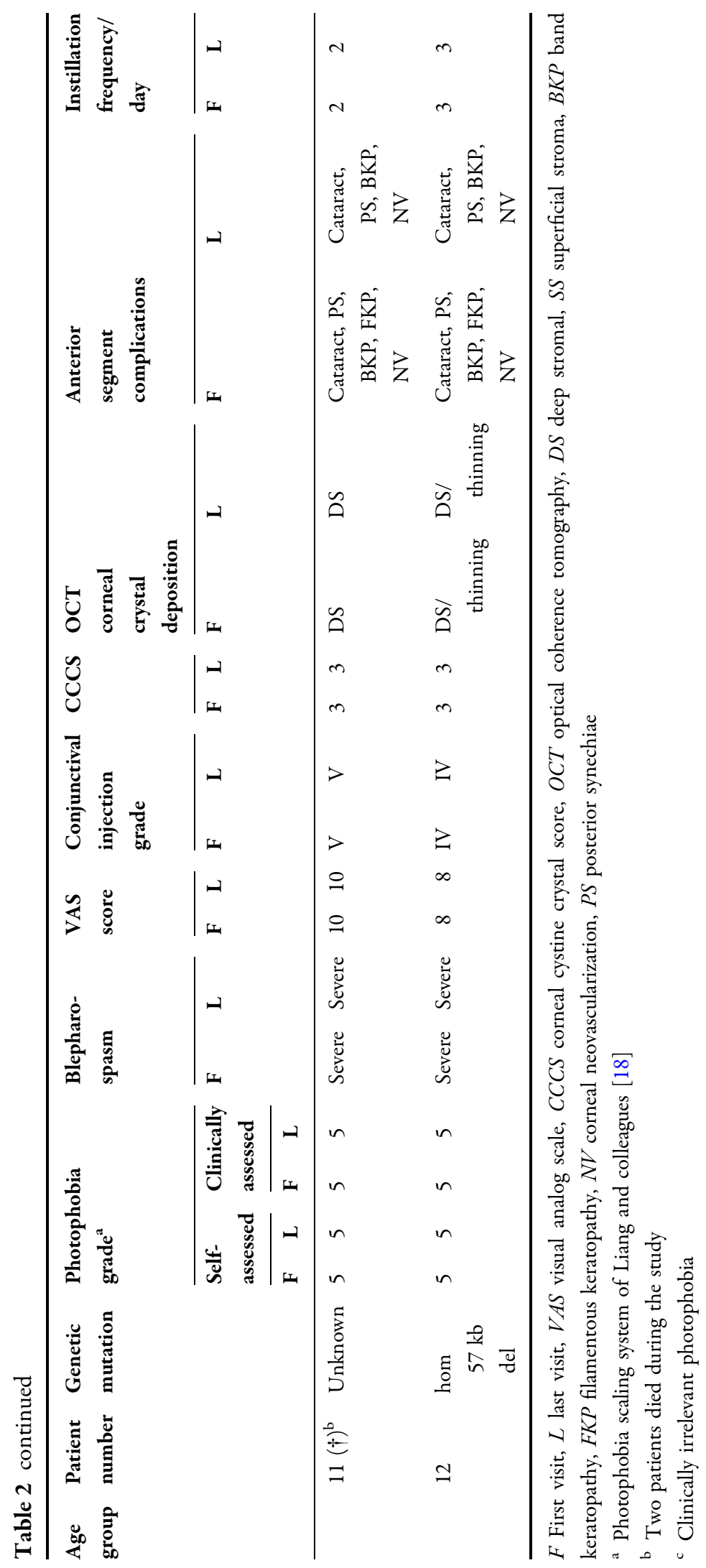

么 Adis 


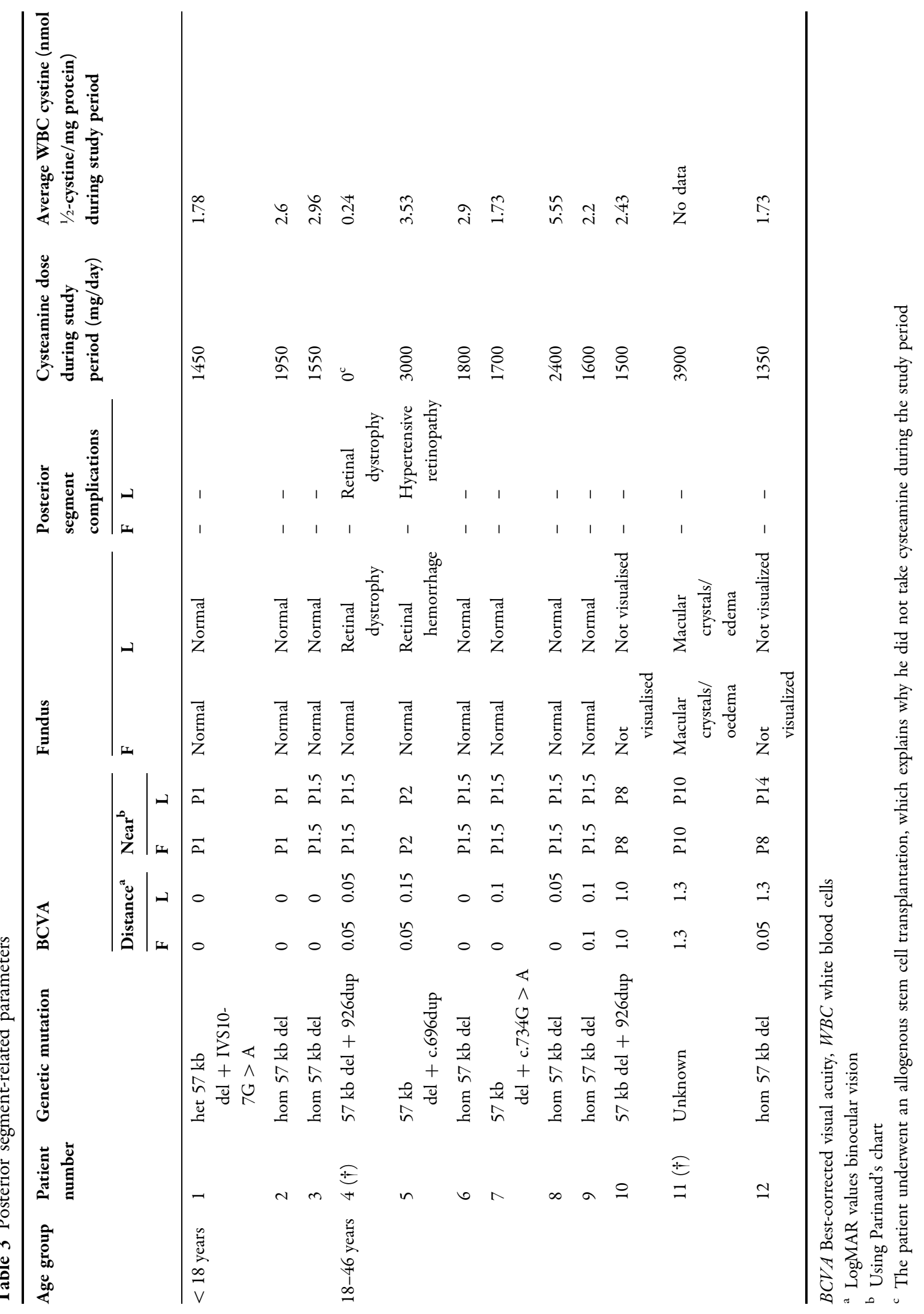




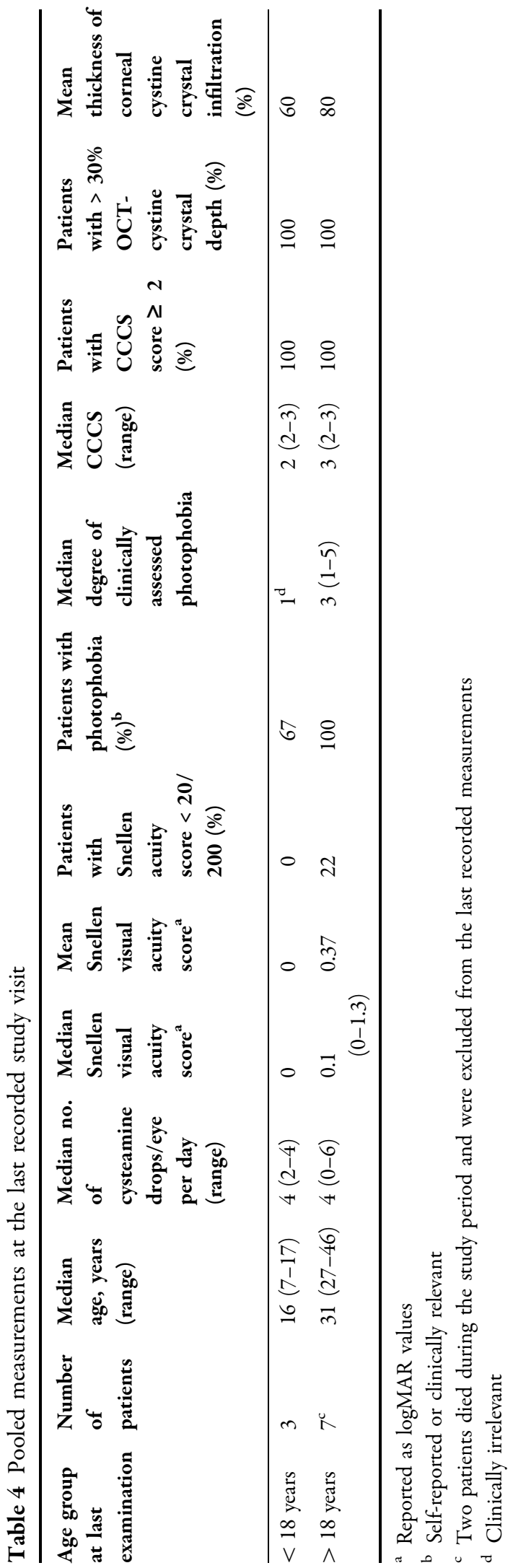

is affected [8]. The crystals typically cause progressive photophobia and subsequent blepharospasm, impairing daily activities. Corneal punctate epithelial erosions, filamentary keratopathy, erosions, peripheral vascularization, band keratopathy, decreased corneal sensitivity, increased corneal thickness and glare have also been reported [8-20]. Corneal crystals do not necessarily cause a decrease in visual acuity; however, pigmentary retinopathy can be associated with the accumulation of these crystals in the cornea [21]. All patients included in this case study showed a higher CCCS $(\geq 2)$ at the end of the study, indicating progressive cystine depositions in the cornea despite treatment with aqueous $0.5 \%$ cysteamine eye drops. No improvement was observed in the degree of photophobia, blepharospasm and anterior segment complications. These results indicate that treatment with a compounded preparation of aqueous $0.5 \%$ cysteamine eye drops was not effective in this patient population in reducing the ocular symptoms of cystinosis at the doses used.

While corneal crystals can be found in all patients with cystinosis, retinal pathology, in contrast, is not always present [8]. Bilateral and relatively symmetrical peripheral patches of depigmentation and mottling can be detected in the temporal quadrants; these progress to the posterior pole with macular edema, resulting in a decrease in visual acuity $[8,20]$. Oral cysteamine treatment has a favorable effect on the retinal complications, whereas topical therapy is not effective in preventing them. In our case series, retinal dystrophy and retinal hemorrhages were observed in one patient.

Cysteamine eye drops have been reported to be beneficial in the treatment of corneal cystine accumulation and symptoms of keratopathy $[7,14]$ and are recommended to be used frequently, preferably every waking hour [16]. The compounded preparation used in our study is comparable to the standard formulation described in the study by Tsilou et al. [11]. The difference in efficacy could be explained by the suboptimal dosage regimen in our patients. Other formulations with comparable or lower concentrations of cysteamine have also been described to be successful at an hourly regimen 
[12-15]. Consequently, hourly instillation would seem to be more important than the concentration of cysteamine. However, this recommended dosage is not useful in real-life situations. Furthermore, treatment with cysteamine eye drops is reported to be associated with a burning and stinging sensation $[3,11]$, which might explain the reduced compliance $(<6$ times/day) observed in patients included in this study.

Although cysteamine eye drops are the only available topical treatment in ocular cystinosis, these eye drops are not commercially available in Belgium, in contrast to most European countries. To counter this problem, hospital pharmacies usually prepare and dispense these off-licensed drops. However, these compounded cysteamine eye drops are not stable at different storage conditions. Compounded cysteamine eye drops can be easily oxidized to a less effective cystamine when stored at temperatures above $-20^{\circ} \mathrm{C}[22,23]$, which may negatively affect the efficacy of cysteamine eye drops. This instability may provide an explanation of why we found limited evidence of effectiveness in the patients included in this retrospective case study. However, since the stability of our formulation was not tested in this study, we cannot confirm this hypothesis at this time.

Cystadrops ${ }^{\circledR}$ (Recordati Rare Diseases, Milan, Italy) is a recently approved gel-like viscous commercial preparation of cysteamine that is indicated for the treatment of corneal cystine crystals in patients with cystinosis who are older than 2 years [24]. Clinical trials have confirmed its efficacy and safety in this target patient population $[25,26]$. The recommended dose of Cystadrops ${ }^{\circledR}$ is four times a day during waking hours [24] compared with once every waking hour for compounded cysteamine eye drops. Cystadrops ${ }^{\circledR}$ is also a more stable preparation of cysteamine and can be stored at room temperature for 7 days after opening [24]. The decreased dosing frequency and improved stability of Cystadrops ${ }^{\circledR}$ may favor its use in the treatment of ocular cystinosis.

The main limitation of the present study is that it is a retrospective review of patients attending a single multidisciplinary clinic; consequently, it is subject to selection bias. In addition, some parameters reported by patients (e.g. pain, photophobia) were subjective assessments, and there was no quantification of corneal cystine deposition by means of in vivo confocal microscopy due to this procedure not being availability to the investigators during the study period. No control group was included, so the therapeutic impact of cysteamine drops could not be assessed. Also, the lack of efficacy of cysteamine eye drops seen in the study patients may be due to the low frequency of instillation and the relatively short duration of follow-up.

\section{CONCLUSION}

In conclusion, this retrospective study of cases of cystinosis showed that despite treatment with a compounded preparation of aqueous $0.5 \%$ cysteamine eye drops in a real-world situation, corneal complications continued to deteriorate over time in patients with cystinosis, highlighting the need for more stable formulations of cysteamine eye drops.

\section{ACKNOWLEDGEMENTS}

Funding. This study and the Rapid Service Fees were funded by Recordati Rare Diseases. Elena Levtchenko has consultancy agreement with Recordati Rare Diseases, Chiesi and was supported by a research grant of Horizon Pharma.

Medical Writing and Editorial Assistance. We would like to thank Nishad Parkar, $\mathrm{PhD}$, of Springer Healthcare Communications for writing the outline and the subsequent drafts of this manuscript. This medical writing assistance was funded by Recordati Rare Diseases.

Authorship. All named authors meet the International Committee of Medical Journal Editors (ICMJE) criteria for authorship for this article, take responsibility for the integrity of the work as a whole, and have given their approval for this version to be published. 
Author Contributions. IC participated in the clinical follow-up of patients included in this case report and was involved in the data compilation from follow-up visits. IC was also involved in reviewing the manuscript drafts, providing inputs and approving the final draft of the manuscript before submission. FP was involved in reviewing the manuscript drafts, providing input and finishing the final draft of the manuscript before submission. KV provided the genetic data for the patients. EL, KV, KVK and CC were involved in approving the final draft of the manuscript before submission.

Disclosures. Freya Peeters, Catherine Cassiman, Karel Van Keer, Elena Levtchenko, Koenraad Veys and Ingele Casteels have nothing to disclose.

Compliance with Ethics Guidelines. The Ethical committee of the University Hospitals Leuven was contacted concerning this publication. Since all data had been previously acquired for other purposes and it concerned a retrospective analysis, no further approval or consent was required.

Data Availability. All data generated or analyzed during this study are included in this published article/as supplementary information files.

Open Access. This article is distributed under the terms of the Creative Commons Attribution-NonCommercial 4.0 International License (http://creativecommons.org/licenses/ by-nc/4.0/), which permits any noncommercial use, distribution, and reproduction in any medium, provided you give appropriate credit to the original author(s) and the source, provide a link to the Creative Commons license, and indicate if changes were made.

\section{REFERENCES}

1. Gahl WA, Thoene JG, Schneider JA. Cystinosis. N Engl J Med. 2002;347(2):111-2. https://doi.org/ $10.1056 /$ nejmra020552.
2. Nesterova G, Gahl WA. Cystinosis: the evolution of a treatable disease. Pediatr Nephrol. 2013;28(1):51-9. https://doi.org/10.1007/s00467012-2242-5.

3. Emma F, Nesterova G, Langman C, et al. Nephropathic cystinosis: an international consensus document. Nephrol Dial Transp. 2014;29[Suppl 4]:iv87-94. https://doi.org/10.1093/ndt/gfu090.

4. Elmonem MA, Veys KR, Soliman NA, et al. Cystinosis: a review. Orphanet J Rare Dis. 2016;11(1):1. https://doi.org/10.1186/s13023-016-0426-y.

5. Gahl WA. Cystinosis. In: Avner E, Harmon W, Niaudet $P$, Yoshikawa N, editors. Pediatric nephrology. Berlin: Springer; 2009. p. 1019-38.

6. Al-Haggar M. Cystinosis as a lysosomal storage disease with multiple mutant alleles: phenotypicgenotypic correlations. World J Neprhol. 2013;4(2):94-102. http://www.wjgnet.com/22206124/full/v2/i4/94.htm.

7. Gahl WA, Kuehl EM, Iwata F, et al. Corneal crystals in nephropathic cystinosis: natural history and treatment with cysteamine eyedrops. Mol Genet Metab. 2000;71(1-2):100-2. https://doi.org/10. 1006/mgme.2000.3062.

8. Tsilou E, Zhou M, Gahl W, et al. Ophthalmic manifestations and histopathology of infantile nephropathic cystinosis: report of a case and review of the literature. Surv Ophthalmol. 2007;52(1):97-105. https://doi.org/10.1016/j. survophthal.2006.10.006.

9. Gahl WA, Tietze F, Butler JD, et al. Cysteamine depletes cystinotic leucocyte granular fractions of cystine by the mechanism of disulphide interchange. Biochem J. 1985;228(3):545-50. https:// doi.org/10.1042/bj2280545.

10. Ariceta G, Giordano V, Santos F. Effects of longterm cysteamine treatment in patients with cystinosis. Pediatr Nephrol. 2019;34(4):571-8. https:// doi.org/10.1007/s00467-017-3856-4.

11. Tsilou ET. A multicentre randomised double masked clinical trial of a new formulation of topical cysteamine for the treatment of corneal cystine crystals in cystinosis. $\mathrm{Br} \mathrm{J}$ Ophthalmol. 2003;87(1):28-31. https://doi.org/10.1136/bjo.87. 1.28 .

12. Bradbury JA, Danjoux J-P, Voller J, et al. A randomised placebo-controlled trial of topical cysteamine therapy in patients with nephropathic cystinosis. Eye. 1991;5(6):755-60. https://doi.org/ 10.1038/eye.1991.139. 
13. Jones NP, Postlethwaite RJ, Noble JL. Clearance of corneal crystals in nephropathic cystinosis by topical cysteamine $0.5 \% \mathrm{Br} \mathrm{J}$ Ophthalmol. 1991;75(5):311-2. https://doi.org/10.1136/bjo.75. 5.311 .

14. Kaiser-Kupfer MI, Fujikawa L, Kuwabara T, et al. Removal of corneal crystals by topical cysteamine in nephropathic cystinosis. $\mathrm{N}$ Eng J Med. 1987;13(316):775-779. http://www.nejm.org/doi/ full/10.1056/NEJM198703263161304?url_ver=Z39. 88-2003\&rfr_id=ori:rid:crossref.org\&rfr_dat $=\mathrm{cr}_{\text {_ }}$ pub\%3dpubmed.

15. MacDonald IM, Noel LP, Minntsioulis G, et al. The effect of topical cysteamine drops on reducing crystal formation within the cornea of patients affected by nephropathic cystinosis. J Pediatr Ophthalmol Strabismus. 1990;5(27):1.

16. US Food and Drug Administration. CystaranTM (cysteamine ophthalmic solution) prescribing information. US Food and Drug Administration. 2012. http://www.accessdata.fda.gov/drugsatfda docs/label/2012/200740s000lbl.pdf.

17. Pinxten A-M, Hua M-T, Simpson J, et al. Clinical practice: a proposed standardized ophthalmological assessment for patients with cystinosis. Ophthalmol Ther. 2017;6(1):93-104. https://doi.org/10. 1007/s40123-017-0089-3.

18. Liang H, Baudouin C, Hassani R, et al. Photophobia and corneal crystal density in nephropathic cystinosis: an in vivo confocal microscopy and anteriorsegment optical coherence tomography study. Invest Ophthalmol Vis Sci. 2015;56(5):3218. https://doi.org/10.1167/iovs.15-16499.

19. Terry RL, Schnider CM, Holden BA. CCLRU standards for success of daily and extended wear contact lenses. Optom Vis Sci. 1993;3(70):234-43.
20. Flockerzi E, Daas L, Schlötzer-Schrehardt U, et al. Ocular changes in nephropathic cystinosis: the course of the gold-dust. Int Ophthalmol. 2019;39(6):1413-8. https://doi.org/10.1007/ s10792-018-0954-7.

21. Wong VG, Lietman PS, Seegmiller JE. Alterations of pigment epithelium in cystinosis. Arch Ophthalmol. 1967;77(3):361-9. https://doi.org/10.1001/ archopht.1967.00980020363014.

22. Reda A, Van Schepdael A, Adams E, et al. Effect of storage conditions on stability of ophthalmological compounded cysteamine eye drops. JMID Rep. 2017;42:47-51. https://doi.org/10.1007/8904_ 2017_77.

23. Iwata F, Kuehl EM, Reed GF, et al. A randomized clinical trial of topical cysteamine disulfide (cystamine) versus free thiol (cysteamine) in the treatment of corneal cystine crystals in cystinosis. Mol Genet Metab. 1998;64(4):237-42. https://doi.org/ 10.1006/mgme.1998.2725.

24. European Medical Agency. Cystadrops ${ }^{\circledR}$ Summary of product characteristics. 2017. http://www.ema. europa.eu/documents/product-information/ cystadrops-epar-product-information_en.pdf.

25. Labbé A, Baudouin C, Deschênes G, et al. A new gel formulation of topical cysteamine for the treatment of corneal cystine crystals in cystinosis: the Cystadrops OCT-1 study. Mol Genet Metab. 2014;111(3):314-20. https://doi.org/10.1016/j. ymgme.2013.12.298.

26. Liang H, Labbé A, Le Mouhaër J, et al. A new viscous cysteamine eye drops treatment for ophthalmic cystinosis: an open-label randomized comparative phase III pivotal study. Invest Ophthalmol Vis Sci. 2017;58(4):2275. https://doi.org/10.1167/iovs.1621080. 